\title{
Review
}

\section{Future possibilities in the prevention of breast cancer Fat and fiber and breast cancer research} Ross L Prentice

\author{
Fred Hutchinson Cancer Research Center, Seattle, Washington, USA
}

Received: 25 November 1999

Accepted: 30 March 2000

Published: 19 May 2000
Breast Cancer Res 2000, 2:268-276

(c) Current Science Ltd

\begin{abstract}
The potential for a reduction in dietary fat or for an increase in dietary fiber to reduce breast cancer risk has been debated for some years. It is argued here that available research data, even though extensive, leave open hypotheses ranging from little or no potential to major public health potential for breast cancer prevention by means of these dietary maneuvers. Some elements of a research strategy for testing these and other dietary breast cancer prevention hypotheses are described.
\end{abstract}

Keywords: breast cancer, fat, fiber, measurement error, research strategies

\section{Introduction}

The hypothesis that a reduction in dietary fat in Western populations will lead to reductions in breast cancer risk has been promulgated for some decades. Its evaluation has been the goal of considerable research, and subject to considerable controversy (for example $\left[1^{\circ}, 2^{\circ}\right]$ ). In effect, the testing of this hypothesis has become a prominent test case in a debate over the study designs and research strategies that are needed to obtain reliable information in the broader research area of diet and chronic disease. On the one hand, some evidently believe that reliable information on the fat and breast cancer relationship can be based on self-reported diet in the context of observational studies. This perspective led investigators involved in a recent pooled analysis of cohort studies of fat consumption and breast cancer risk [ $\left.3^{\circ}\right]$ to conclude that 'In the context of the Western lifestyle, lowering the total intake of fat in midlife is unlikely to reduce the risk of breast cancer substantially.' On the other hand, the US National Institutes of Health-sponsored Women's Health Initiative clinical trial $\left[4^{\circ}\right]$ is about half way to completion. This Initiative includes a randomized controlled clinical trial, one component of which is to assess the impact of a 'low-fat-eating pattern' on the incidence of breast cancer and colorectal cancer (and coronary heart disease secondarily) among 48837 postmenopausal women in the USA. The existence of this large, complex intervention trial reflects the view of others that the hypothesis that a low-fat-eating pattern helps to prevent breast cancer is worth testing, and that observational studies alone may not be able to provide sufficiently reliable information on the fat and breast cancer association, regardless of their size or duration.

The hypothesis that the increased consumption of dietary fiber may reduce breast cancer risk has also existed for some decades. The relationship between estimated fiber consumption and breast cancer risk has been examined in a number of observational studies $\left[5^{\circ}\right]$. Also, the low-fateating pattern targeted in the Women's Health Initiative clinical trial $\left.4^{\circ}\right]$ includes modest increases in fruit and vegetable consumption, and in grain consumption, in addition to its major reduction in percentage of energy from fat from customary levels of about $35 \%$ to a targeted $20 \%$.

Various meta-analyses and reviews of studies pertinent to these hypotheses have been published, precluding the 
need for a study-by-study review here. The reported observational study associations of fat and fiber with breast cancer appear to be inconsistent among study designs, however, contributing to the previously mentioned controversy. The focus of this review, then, is on the strengths, weaknesses and interpretation of available data, and on aspects of a research agenda that may help to bring clarity to this important area, and clarity to the assessment of other diet, nutrition, and chronic disease prevention hypotheses.

\section{Hypothesis generation}

Experimental studies in rodents dating back to the $1940 \mathrm{~s}$ [6] pointed to the ability of a high-fat diet to promote the development of mammary tumors, with the possibility that certain polyunsaturated fats may be particularly effective promoters [7]. A question ensued [8] as to whether fat specifically, or energy more generally, explained such promotion. A meta-analysis [ $9^{\circ}$ ] of data from rodent feeding studies confirmed a substantial role for calorie restriction in inhibiting mammary tumorigenesis, but also revealed an important role for fat reduction beyond that attributable to the corresponding energy reduction.

Beginning in the 1960 s various groups [10-12,13 ${ }^{\circ}$ examined the relationship between national estimates of the per capita supply of fat and the corresponding rates of breast cancer incidence or mortality, or the relationship between changes in per capita fat supply and corresponding changes in breast cancer rates [13*]. These correlational analyses suggest that dietary fat may have an important link to human breast cancer risk. For example, Prentice and Sheppard [13 ${ }^{\circ}$ presented regression analyses of breast cancer incidence rates on per capita fat supply using data from 21 countries worldwide that have nationally representative cancer incidence data. These analyses suggest that a 50\% reduction in fat consumption in the USA could eventually lead to a breast cancer risk reduction by a factor of about 0.39 among women in the age range 55-69 years and by a factor of about 0.53 among women in the 30-44 years age range. Moreover, the supply of nonfat calories did not contribute further to an explanation for the variations in international breast cancer rates, and neither did per capita gross national product, as a general potential confounding factor related to national development. Very similar relative risk estimates arose from analyses in a subset of these countries that regressed changes in breast cancer incidence rates on corresponding changes in per capita fat supply from a time point about 5 years earlier [13 ${ }^{\circ}$.

Migrant studies have considerable potential to contribute to fat and breast cancer hypothesis generation, but few such studies have included pertinent dietary data. Nonetheless, the substantial breast cancer case-control

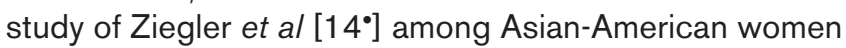
of age 55 years or younger pointed to the '... crucial importance of lifestyle and environment in the etiology of this disease.' In fact those investigators observed a sixfold gradient in breast cancer risk across migration patterns. Among migrants with a single move from East to West, the relative risk estimates were $0.32,0.40,0.72$, 0.66 and 0.59 for $2-4,5-7,8-14,15-21$, and $\geq 22$ years lived in the West, respectively, as compared with women who had always lived in the West, suggesting noteworthy changes in risk within the first decade after migration. Moreover, those investigators did not detect a trend in risk as a function of age at migration through 35 years of age, suggesting that recent exposures may be particularly relevant to breast cancer risk. Exposures that may contribute to this sixfold relative risk trend include hormonal, anthropometric and reproductive factors, most of which are diet related to some extent.

The compelling evidence that circulating estrogens influence breast cancer risk [15-18] has prompted study of the influence of dietary intervention on endogenous estrogen levels. Wu et al [19'] recently reported a meta-analysis of 13 intervention trials that examined changes in estradiol levels subsequent to dietary fat reduction. Those investigators calculated estradiol reductions (95\% confidence intervals) of $-7.4 \%(-11.7$ to $-2.9 \%)$ among premenopausal women and $-23.0 \%(-27.7$ to $-18.1 \%)$ among postmenopausal women. The greatest estradiol reductions occurred in two studies in which dietary fat was reduced to $10-12 \%$ of calories, but significant reductions remained when the analysis was restricted to the remaining studies, which had energy from fat in the range of $18-25 \%$. Some of the dietary interventions studied involved increased fiber in addition to fat reduction, but reductions in estradiol levels were found in studies with low, medium, and high fiber intake. Other studies $[20,21]$ have shown reductions in blood estradiol with increases in fiber intake without change in percentage energy from fat. Any such reduction, however, may depend on the type of fiber; for example, wheat bran versus oat or corn bran, and on fiber solubility.

Although the effects of dietary intervention on blood estrogens are of considerable interest, they do not allow one to assert a corresponding effect of dietary intervention on breast cancer in view of the many other biological effects that may result from dietary change. Also, the intervention trials that examined these changes in exogenous estrogens tended to be of, at most, a few months in duration, during which the women studied may have experienced some weight loss, reducing the ability to project dietary intervention effects on hormone concentrations in the longer term.

\section{Association studies}

The conduct of analytic epidemiologic studies to relate individual nutrient consumption to corresponding breast cancer 
Table 1

Summary relative risk estimates from observational studies of fat intake* and breast cancer risk, along with corresponding projections from international correlational analyses

\begin{tabular}{|c|c|c|c|c|c|c|}
\hline \multirow[b]{2}{*}{ Data source } & \multicolumn{5}{|c|}{ Total fat consumption quintile } & \multirow[b]{2}{*}{ Trend test } \\
\hline & $1^{+}$ & 2 & 3 & 4 & 5 & \\
\hline Case-control studies $\left[5^{*}\right]$ & 1.00 & 1.20 & 1.24 & 1.24 & 1.46 & $P=0.0002$ \\
\hline \multirow[t]{2}{*}{ International data projection $\left[23^{\star}\right]$} & 1.00 & 1.11 & 1.19 & 1.28 & 1.42 & \\
\hline & \multicolumn{5}{|c|}{ Calorie-adjusted fat intake quintile } & \\
\hline Data source & $1^{+}$ & 2 & 3 & 4 & 5 & Trend test \\
\hline Cohort studies $\left[3^{\star}\right]$ & 1.00 & 1.01 & 1.12 & 1.07 & 1.05 & $P=0.21$ \\
\hline International data projection $\left[23^{\star}\right]$ & 1.00 & 1.07 & 1.12 & 1.19 & 1.27 & \\
\hline
\end{tabular}

*Most of the case-control studies and all cohort studies used a food frequency assessment of diet; international data projections include an accommodation of food frequency measurement error. ${ }^{\dagger}$ Reference category.

occurrence constitutes a logical step in the evaluation of the fat, fiber, and breast cancer hypotheses. There have been many such studies during the past 25 years. Initially most were case-control studies, but by now there are several large cohort studies as well. Although studies of these types could attempt to examine reductions in fat and increases in fiber in relation to breast cancer risk, perhaps using migrant populations, to date essentially all have chosen to associate current or recent nutrient intake with breast cancer risk. Furthermore, all have relied upon self-reported food intake, often in the form of food frequency data in conjunction with a nutrient database, to estimate each individual's consumption of fat, fiber, and other nutrients.

Unfortunately there are substantial obstacles to finding clear and interpretable relationships in this type of study for several reasons. In increasing order of importance, these include the following.

1. Current or recent nutrient intakes may differ from those over the years or decades pertinent to breast cancer risk, likely attenuating any fat, fiber, and breast cancer association.

2. Certain nutrient intakes in Western populations may not be highly variable, despite the variety of foods available; for example, at present the vast majority of Americans probably have dietary energy from fat in the range $27-40 \%$, resulting in modest relative risk gradients within study populations, even if the nutrient exposures are important risk factors.

3. The diet is a complex mixture of foods and nutrients including many highly correlated elements; even if the nutrient exposures of interest could be measured exactly, it may be a formidable task to estimate the relationship between a specific nutrient, such as fat or fiber, and breast cancer risk, while accommodating other dietary factors.
4. Dietary patterns may relate in a complicated manner to other breast cancer risk factors, including anthropometric measures, reproductive factors and physical activity patterns; hence, there is potential for confounding (or over-control), which may be unavoidable for such difficult-to-measure exposures as physical activity patterns.

5. Key measurement properties of existing dietary selfreport instruments are unknown, even in the populations in which these instruments have been applied.

There have been a number of meta-analyses of the published fat and breast cancer association studies. For a fairly recent example, Boyd et al [22] calculated a summary relative risk estimate (95\% confidence interval) of 1.12 (1.04-1.21) for the highest versus lowest fat intake categories used in the reporting of 23 epidemiologic studies, including 16 case-control and seven cohort studies. However, the summary relative risk estimate from the case-control studies was $1.21(1.10-1.34)$, which is quite different from that (1.01 [0.90-1.13]) from the cohort studies.

It is fortunate that the individual level data from most of these studies have been assembled for standardized analyses. Howe et al $\left[^{\circ}{ }^{\circ}\right.$ carried out a combined analysis of 12 case-control studies involving 4427 breast cancer cases and 6095 control individuals, about two-thirds of whom were postmenopausal. Table 1 shows their summary postmenopausal breast cancer relative risk estimates across the total fat intake categories defined by the quintiles of one of the Canadian case-control studies. A highly significant trend $(P=0.0002)$ was reported. The relative risk trend was much less pronounced among premenopausal women and was not statistically significant $(P=0.21)$. Table 1 also shows relative risks projected 
from the international correlational analyses. These projections $\left[23^{\circ}\right]$ are based on a regression of log-breast cancer incidence in the age range 55-69 years on log-total fat, and assume a classical measurement model for food frequency and 4-day food record assessments of log-fat intake. The projections actually apply to the intake quintiles of the baseline food frequency data in the Women's Health Trial [24], which differ little from those from the Canadian study quintiles used by Howe et al [5]. A close correspondence between the case-control and the international data estimates can be seen. Howe et al commented further that there was no evidence that nonfat sources of energy were associated with breast cancer risk, after allowing for total fat, in their analyses.

Hunter et al [3०] provided a pooled analysis of seven cohort studies including 4980 breast cancer cases out of 337819 women. Summary relative risk estimates were presented across quintiles of calorie-adjusted fat intake, based on food frequency dietary assessments, and are shown in Table 1. The breast cancer risk did not show a significant trend $(P=0.21)$ across intake categories. Table 1 also shows corresponding relative risk projections from international correlational analyses, again with measurement error acknowledged using a classical measurement model. The projection categories are based on baseline food frequency percentage energy from fat from the Women's Health Trial, which are likely to differ little from the calorie-adjusted fat categories used in the pooled cohort analyses. The projections are based on international breast cancer rates for the age range 55-69 years, so that these could be reduced somewhat to acknowledge the inclusion of premenopausal women in the pooled cohort analyses. Note the difference between observed and projected relative risks in the upper categories of calorie-adjusted fat intake.

In fact, the relative risk estimates and projections shown in Table 1 apparently capture the basis for the controversy over the fat and breast cancer hypothesis. Population scientists are accustomed to viewing cohort studies as the most reliable of the observational study strategies. It can therefore be argued (for example $\left[2^{\circ}, 3^{\circ}\right]$ ) that dietary fat reduction is unlikely to reduce breast cancer risk meaningfully among middle-aged and older women, on the basis of the following factors: the cohort study lack of association noted in Table 1; the possibility of selection or recall biases in the case-control studies $[25,26]$; a lack of ability to control confounding in international correlational analysis; a lack of clarity of implications of low-fat intervention effects on endogenous hormones; and a lack of direct relevance of animal feeding experiments.

On the other hand one can regard these data sources as supportive of an important fat and breast cancer relationship, and can question the extent to which the cohort analyses provide contrary evidence. Certainly the relative risk trend projected from international correlational analyses (Table 1) is modest enough to be well within the range that may not be reliably detected in observational studies, regardless of their size, on the basis of the reasons listed above. Among these reasons, the impact of measurement error in dietary assessment is the least well understood, and is most crucial to the interpretation of both the cohort and case-control studies. Often in the reporting of these studies the authors will write words to the effect that analyses were conducted that correct for measurement

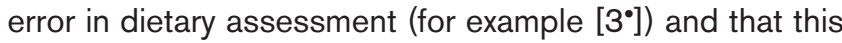
correction did not alter study findings. It is important to understand the assumptions that underlie such measurement error adjustments.

Available measurement error methods require a nutrient consumption assessment that estimates the quantity of interest aside from an additive error that must be independent of the targeted quantity and of other study subject characteristics. To be specific, let $Z$ represent a woman's average daily total fat intake (or percent energy from fat) over a time period pertinent to breast cancer risk (eg the preceding decade). One needs to assume that an estimate $\left(X_{1}\right)$ is available, at least on a subsample of the study population, such that:

$$
X_{1}=Z+e_{1}
$$

Where the error term ' $e_{1}$ ' is assumed to be independent of the 'true' intake $Z$, so that women with high fat consumption are no more likely to underestimate their fat intake than are women consuming less fat. Also the measurement error $e_{1}$ is typically assumed to be independent of such study subject characteristics or exposures as ethnicity, age, physical activity patterns or body mass. These assumptions are typically applied to data from food records or recalls collected on a small subsample of study subjects. Self-report data using a less expensive and less comprehensive food frequency questionnaire are obtained for all study subjects. The most flexible measurement model for the food frequency estimate of fat that has been used in these studies supposes that the food frequency estimate ' $X{ }_{2}$ ' can be written:

$$
X_{2}=Z^{*}+e_{2}
$$

where ' $Z^{*}$ ' is a variable that is positively correlated with $Z$, and where the error term ' $e_{2}$ ' is independent of $Z^{*}$ and independent of other study subject characteristics and, importantly, is independent of the error $e_{1}$. These assumptions allow $X_{1}$ to be used to 'calibrate' the food frequency measure, in that $X_{2}$ can be replaced by an estimate of $Z$ given $X_{2}$ in relative-risk analysis.

These regression calibration methods (as described for example in [27]) work well when the measurement error is 
not too large and the assumptions listed above hold. In the diet and chronic disease area, however, the measurement errors are not small, as can be seen by observing the modest correlations between nutrient intake estimates from multiple self-report instruments at a single point in time (eg about 0.3 for total fat or 0.5 for calorie-adjusted fat), or between repeat applications of the same instrument at different time points (eg in [28]). Furthermore, there are reasons to suspect strongly that the assumptions that underlie Equations 1 and 2 do not hold for selfreported fat intake.

For example, the self-reporting of dietary habits is thought to depend on social desirability factors $[29,30]$, suggesting that it is unlikely that obese and slim women would have the same measurement error reporting characteristics. The absence of an accepted biomarker of fat intake precludes direct study of the measurement errors in Equations 1 and 2. However, doubly labeled water studies of short-term energy expenditure indicate energy consumption to be substantially under-reported, in the range $25-50 \%$, among obese women with little or no underreporting among slim women [31 ${ }^{\circ}$, making it highly probably that fat calories are also greatly under-reported by obese women. Moreover, protein expenditure, as assessed by urinary nitrogen, appeared to be underreported to a lesser extent than total energy [31 ${ }^{\circ}$, making it plausible that percentage energy from fat is also substantially under-reported by obese women.

These data indicate that energy or fat intakes from food records (or recalls) do not conform to the assumptions that underlie Equation 1, and hence do not serve as a suitable 'anchor' for calibrating the corresponding food frequency data. Another implication of these data is that measurement errors from food record (Eqn 1) and food frequency (Eqn 2) assessments of fat intake, or calorie adjusted fat intake, may well be positively correlated. For example, an obese woman who underestimates her per capita fat consumption by, say, $50 \%$ on food records may also systematically underestimate her fat consumption on a food frequency questionnaire. In this case even the modest correlations (eg 0.3) previously noted between food record and food frequency fat consumption may be due in whole or in part to correlated measurement error, rather than to an ability of these dietary instruments to measure actual fat consumption.

Prentice $\left[23^{\circ}\right]$ carried out some exercises under which the food record and food frequency estimates of total fat, or percentage energy from fat, were allowed to have correlated measurement errors. The international data projected relative risks were very sensitive to such correlation, in that even the modest trends shown in Table 1 could readily be further reduced or distorted. This type of exercise implies that it is necessary to have rather firm information on the measurement characteristics of the food frequency questionnaires in the context of the cohort or case-control studies in order to determine whether the relative risk trends shown in Table 1 differ appreciably from expectation under the generating hypothesis. In other words, one can take the point of view, with the current state of knowledge of the properties of dietary assessment instruments, that cohort studies [ $\left.3^{\circ}\right]$, in addition to other observational studies, are limited to the extent that even the question of breast cancer association with fat, or percentage of energy from fat, has yet to be answered, let alone the question of fat reduction and breast cancer prevention.

It would seem to be a fact, even after 25 years of data collection and deliberation, that well-intentioned scientists can hold diverse views ranging from the lack of any meaningful potential for a fat reduction to reduce breast cancer risk, to major public health potential, without compelling contradiction from available data. This state of affairs is a testimony to the real methodologic difficulties that pervade this research area.

A number of the observational studies commented on the association between the intake of specific types of fat and breast cancer risk. For example, Howe et al [5 ${ }^{\circ}$ ] observed the strongest trends in breast cancer risk with estimated

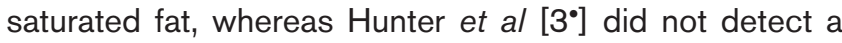
significant trend with any of energy-adjusted saturated, monounsaturated or polyunsaturated fats. In comparison, animal experiments [7] and international correlational analyses $\left[13^{\circ}\right]$ suggest a role for saturated fat, along with a particularly important role for polyunsaturated fat. None of these data can be viewed as compelling for human cancer. In particular, sorting out associations with specific sources of fat in the context of cohort and case-control studies would evidently require exquisite knowledge of the joint measurement characteristics of the assessment instruments with regard to these intakes and the consumption of energy.

The fiber and breast cancer association has received much less attention. Howe et al [5ं] reported a significant $(P=0.002)$ association of estimated dietary fiber and breast cancer risk among postmenopausal women. The association among premenopausal women was similar, but not significant $(P=0.15)$. These analyses were based on data from the same 12 case-control studies, and included an adjustment for total fat intake. However, when $\beta$-carotene and vitamin $\mathrm{C}$ were added to the postmenopausal breast cancer regression analysis, fiber ceased to be significantly related to breast cancer risk $(P=0.78)$, whereas vitamin $\mathrm{C}$ consumption remained significant. This suggests that the marginal association of breast cancer with fiber intake may be attributable to vitamin $\mathrm{C}$ consumption, or to fruit and vegetable consumption more generally, for which some modest evidence for 
an inverse association exists [32]. On the other hand, selfreported fiber also involves substantial measurement error. For example, Willett et al [28] reported food frequency and food record correlations of 0.43 for crude fiber, and 0.51 for crude fiber adjusted for energy. Hence, any relative risk trends across fiber consumption categories in observational studies are likely to be much attenuated or distorted, depending on the specific measurement properties of the assessment tools and the joint measurement properties of fiber, fat, energy and pertinent micronutrients. As with fat consumption, objective information on the measurement properties of self-reported fiber consumption, particularly for food frequency assessments, seems a prerequisite to the interpretation of observational study breast cancer relative risk associations. Note also that dietary fiber rather than crude fiber is the preferred fiber assay for nutrition studies, and that dietary and crude fiber may have little or no correlation in specific populations.

\section{Intervention trials}

There are four randomized controlled intervention trials currently ongoing that are pertinent to breast cancer prevention hypotheses by means of fat reduction or fiber increases. None have reported breast cancer occurrence or recurrence data as yet. Two of these studies aim to prevent breast cancer recurrence or new primary cancers among women with early or intermediate stage breast cancer. The Women's Intervention Nutrition Study [33] targets a major reduction in dietary fat, whereas the Women's Healthy Eating and Lifestyle Study [34] emphasizes a plant-based diet that is high in vegetables and fruit. These studies are both in the course of enroling 2500-3000 women. The other two intervention trials focus on the reduction in breast cancer occurrence among women without a personal history of breast cancer. The Canadian trial of Boyd et al [35] is studying the impact of a low-fat-eating pattern on breast cancer incidence among approximately 9500 premenopausal and postmenopausal women who are at elevated risk for breast cancer on the basis of mammographic dysplasia.

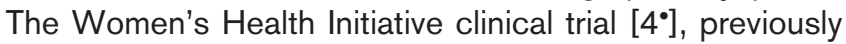
mentioned, is studying the effect of a low-fat-eating pattern on the occurrence of breast cancer and selected other diseases among 48837 postmenopausal women in the USA.

These intervention trials have a number of desirable features. First, by virtue of the randomized assignment to intervention or control status, baseline risk factors, whether recognized or not, are statistically independent of intervention assignment, eliminating the problem of confounding by prerandomization factors. Second, the comparison of breast cancer rates between intervention and control women does not involve individual dietary assessment. Third, intervention trials, by introducing a dietary change, have potential to identify practical preventive maneuvers directly. On the other hand, intervention trials tend to be expensive and logistically difficult, and the maintenance of dietary adherence for a sufficient period of time to be able to ascertain clinical outcomes that may be affected beneficially or detrimentally with sufficient precision can be a formidable task. Furthermore, intervention trials by their very nature test a specific intervention or intervention program, so that the effects of specific elements of the intervention may not be able to be reliably studied. For example, the dietary modification component

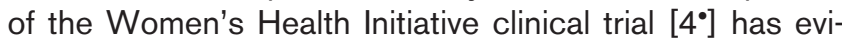
dently resulted in a major reduction in percentage energy from fat and total fat consumption among intervention women, but, consistent with intervention goals, such women also report increases in the consumption of fruit and vegetables and grains. Analyses to relate these specific changes to breast cancer risk reductions may need to rely on individual self-reports of diet, very much like the association studies reviewed in the preceding section. Note, however, that the Women's Health Initiative clinical trial does directly test a dietary pattern of great public health interest.

\section{Future directions and research opportunities}

Measurement properties of the dietary assessment tools used in observational studies play a crucial role in the interpretation of observed relative risk trends and calculation of measurement error-adjusted relative risk trends. Also, self-report assessments of nutrient intakes likely fail to adhere, even approximately, to the measurement error assumptions that attend (Eqn 1). It is therefore natural and important to consider objective measures of nutrient intakes in Equation 1 in future observational studies. For example, the American Association of Retired Persons cohort study, which is being coordinated by the US National Cancer Institute, is incorporating a sizeable substudy that includes doubly labeled water assessments of energy expenditure and urinary nitrogen assessments of protein expenditure. Similarly, our group in Seattle is currently piloting a study of objective measures for a broader range of nutrients, including blood fatty acid measures and various blood micronutrient measures, as a precursor to an intended larger objective measures substudy within the Women's Health Initiative.

The concept behind these efforts is that the biologic assessments of nutrient consumption would play a fundamental role in the analysis and reporting of association studies. Specifically, the available self-report measure $X_{2}$ in Equation 2 would be replaced by an estimate of $Z$ given $X_{2}$ using regression calibration equations.

The measurement properties of the self-report data in application to a specific population may depend on various study subject characteristics, possibly including body mass, ethnicity and age. It may therefore be neces- 
sary for the objective measures substudy to be fairly large, perhaps in the order of 1000-2000 women, for one to be able to calibrate in a sufficiently precise and comprehensive manner. Of course, it may be, as for total fat, that potential biomarkers, including blood fatty acid concentrations and profiles, have yet to yield a recognized measure of dietary consumption, in which case the regression calibration approach will only be able to examine the relationship between breast cancer risk and the quantities targeted by the biomarker. The development of objective measures of the consumption of a broader range of nutrients, and the further study of the methods for the design and conduct of objective measure substudies is an important research goal for strengthening the reliability and interpretability of analytic epidemiologic studies of diet and chronic diseases.

This impact of measurement error in dietary assessment is reduced as the ratio of the measurement error variance to the variance of the nutrient intake decreases. Hence, observational studies that aim to include persons who have an unusually broad range of consumption are also taking a useful step. Ongoing cohort studies that incorporate this aspect include the multinational European Prospective Investigation of Cancer study, the multiethnic Pacific Area Cancer Epidemiology study, and the aforementioned American Association of Retired Persons study, which oversampled persons whose reported fat intakes were in the extremes of the fat consumption distribution. Further observational studies of diet and cancer among migrant populations would also appear to have potential to contribute to this research area.

Students of epidemiology may typically be taught a hierarchy of study reliability, ranging from ecologic studies, case-control studies, cohort studies and intervention trials. In a circumstance of difficult-to-measure, highly correlated exposures and difficult-to-measure confounding factors, however, such a hierarchy may not always hold. In particular, well-conducted ecologic studies in which exposures and potential confounding factors are surveyed among moderate-sized random samples in each group of a multipopulation aggregate study are largely immune to the noise aspect of measurement error $[36,37]$ and may be less sensitive to systematic aspects of measurement error, in part because of the ability to incorporate an unusually broad range of exposures. It is not clear how successfully 'between population' confounding can be controlled in such settings, but the challenges and uncertainties of observational studies in the diet and disease area argue for a varied research program, including research designs that have differing sources of potential bias.

There would appear to be a continuing valuable role for small-scale human feeding studies to further develop and refine the fat, fiber, and breast cancer hypotheses. In par- ticular, it may be that blood estradiol, or blood estrogens more generally, is a sufficiently strong intermediate indicator of breast cancer risk that such studies could reasonably attempt to identify practical dietary patterns that involve reductions in foods containing specific types of fat (eg n-6 fatty acids), or increases in foods that are high in certain types of fiber (wheat, oat or corn bran, legumes, vegetables, fruit) and that may have the ability to reduce estradiol concentrations and to retain such reductions. The situation may be complex, however, in view of the apparent profound effect that estrogen-receptor modulation evidently has in determining breast cancer risk $[38,39]$.

It is my opinion that the types of additional studies alluded to above, in conjunction with the results of ongoing breast cancer occurrence and recurrence intervention trials, can do much to clarify the potential for fat reduction and fiber increase to reduce breast cancer risk. Some prominent epidemiologists evidently believe that the fat and breast cancer question, at least, has already been answered. For example, the keynote speaker at the 1999 annual meeting of the US Society for Epidemiologic Research stated gratuitously that '.. by now, most thinking people have decided that breast cancer is not related to fat intake.' On the other hand, the same speaker has been quoted [40] as stating 'People don't take us seriously anymore, and when they do take us seriously, we may unintentionally do more harm than good.' Indeed, the diet and breast cancer hypotheses, and diet and chronic disease hypotheses more generally, are of such public health importance that they demand our best, integrated effort for their resolution. This is prime time for disease prevention research to receive attention from scientists from a broad range of disciplines. For breast cancer in particular, the next few years may provide the opportunity for lifestyle strategies to be developed that can complement the rapidly developing pharmaceutical strategies in the arsenal of prevention options.

\section{Conclusion}

The hypothesis that a fat reduction or fiber increase will reduce human breast cancer risk remains to be reliably tested, but is of considerable public health importance. Ongoing intervention trials and a diverse program of observational studies have the potential to resolve this and other important diet and chronic disease prevention issues. Though beyond the scope of this review, the new genetic/genomic data that are rapidly becoming available may also help in resolving these issues, for example by illuminating heterogeneity in diet and disease associations, or by facilitating intervention development and testing.

\section{Acknowledgements}

Work by the author and cited in the present review was supported by Public Health Service grant CA53996 from the National Cancer Institute, National Institutes of Health, Department of Health and Human Services. 


\section{References}

Articles of particular interest have been highlighted as:

- of special interest

- of outstanding interest

1. Greenwald P: Role of dietary fat in the causation of breast cancer: - $\quad$ point. Cancer Epidemiol Biomarkers Prev 1999, 8:3-7.

This is a recent summary of arguments in support of a role for dietary fat in causation of breast cancer.

2. Hunter DJ: Role of dietary fat in the causation of breast cancer: - counterpoint. Cancer Epidemiol 1999, 8:9-13.

This is a recent summary of arguments against a role for dietary fat in the causation of breast cancer.

3. Hunter DJ, Spiegelman D, Adami HO, et al: Cohort studies of fat - $\quad$ intake and the risk of breast cancer: a pooled analysis. $N$ Engl $J$ Med 1996, 334:356-361.

A pooled analysis of individual level data from seven cohort studies is presented that concerns the association of self-reported dietary fat and breast cancer risk.

4. Women's Health Initiative Study Group: Design of the Women's - Health Initiative Clinical Trial and Observational Study. Control Clin Trials 1998, 19:61-109.

This is a detailed presentation of the design of the Women's Health Initiative Clinical Trial and Observational Study. The clinical trial includes a randomized controlled evaluation of the ability of a low-fat-eating pattern to reduce the risk of breast cancer and selected other chronic diseases among postmenopausal women.

5. Howe GR, Hirohata T, Hislop TG, et al: Dietary factors and risk of - breast cancer: combined analysis of 12 case-control studies. $J$ Natl Cancer Inst 1990, 82:561-569.

A pooled analysis of individual level data from 12 case-control studies is presented that concerns the association of self-reported dietary fat and dietary fiber and breast cancer risk.

6. Tannenbaum A: Genesis and growth of tumors III. Effects of a high fat diet. Cancer Res 1942, 2:468-475.

7. Newberne PM, Shrager TE, Conner MW: Experimental evidence on the nutritional prevention of cancer. In: Nutrition and Cancer Prevention: Investigating the Role of Macronutrients. Edited by Moon TE, Micozzi MS. New York: Marcel Dekker, 1989:33-82.

8. Albanes D: Total calories, body weight, and tumor incidence in mice. Cancer Res 1987, 47:1987-1992.

9. Freedman L, Clifford C, Messina M: Analysis of dietary, calories, - body weight and the development of mammary tumors in rats and mice: a review. Cancer Res 1990, 50:5710-5719.

This meta-analysis shows a role for energy and for fat specifically in rodent mammary tumorigenesis.

10. Carroll KK, Gammal EB, Plunbett ER: Dietary fat and mammary cancer. Can Med Assoc J 1968, 98:590-594.

11. Armstrong B, Doll R: Environmental factors and cancer incidence and mortality in different countries with special reference to dietary practices. Int J Cancer 1975, 15:617-631.

12. Gray GE, Pike MC, Henderson BE: Breast cancer incidence and mortality rates in different countries in relation to known risk factors and dietary practices. $\mathrm{Br} J$ Cancer 1979, 39:1-7.

13. Prentice RL, Sheppard L: Dietary fat and cancer: consistency of the

- epidemiologic data and disease prevention that may follow from a practical reduction in fat consumption. Cancer Causes Control 1990, 1:81-97.

This is a representative paper on international correlation and time trend analysis of per capita fat supply in relation to the incidence of selected cancers.

14. Ziegler RG, Hoover RN, Pike MC, et al: Migration patterns and

- breast cancer risk in Asian-American women. J Natl Cancer Inst 1993, 85:1819-1827.
This case-control study among Asian-American women demonstrated marked increases in breast cancer risk within a decade of migration to the USA.

15. Pike MC, Spicer DC, Dahmousch L, Press MF: Estrogens, progestogens, normal breast cell proliferation, and breast cancer risk. Epidemiol Rev 1993, 15:17-35.

16. Bernstein L, Ross RK: Endogenous hormones and breast cancer risk. Epidemiol Rev 1993, 15:48-65.

17. Thomas HV, Reeves GK, Key TJ: Endogenous estrogen and postmenopausal breast cancer: a quantitative review. Cancer Causes Control 1997, 8:922-928.

18. Hankinson SE, Willett WC, Manson JE, et al: Plasma sex steroid hormone levels and risk of breast cancer in postmenopausal women. J Natl Cancer Inst 1998, 90:1292-1299.

19. Wu AH, Pike MC, Stramm DO: Meta-analysis: dietary fat intake,

- $\quad$ serum estrogen levels, and risk of breast cancer. J Natl Cancer Inst 1999, 91:529-534.

This meta-analysis of human feeding experiments demonstrated a reduction in blood oestradiol in the weeks after a dietary fat reduction, especially among postmenopausal women.

20. Rose DP, Goldman M, Connolly JM, Strong LE: High-fiber diet reduces serum estrogen concentrations in premenopausal women. Am J Clin Nutr 1991, 54:520-525.

21. Rose DP, Lubin M, Connolly JM: Effects of diet supplementation with wheat bran on serum estrogen levels in the follicular and luteal phases of the menstrual cycle. Nutrition 1997, 13:535-539.

22. Boyd NF, Martin LJ, Noffel M, et al: A meta-analysis of studies of dietary fat and breast cancer risk. Br J Cancer 1993, 68:627-636.

23. Prentice RL: Measurement error and results from analytic epi- demiology: dietary fat and breast cancer. J Natl Cancer Inst 1996, 88:1738-1747.

This paper includes exercises, using dietary data collected in the Women's Health Trial, to indicate that measurement error in self-reported fat or percentage of energy from fat could cause one to miss even an important effect on breast cancer in cohort and case-control studies.

24. Insull W, Henderson MM, Prentice RL, et al: Results of a randomized feasibility study of a low-fat diet. Arch Intern Med 1990, 150:421427.

25. Friedenreich $\mathrm{CM}$, Howe GR, Miller AB: The effect of recall bias on the association of calorie-providing nutrients and breast cancer. Epidemiology 1991, 2:424-429.

26. Giovannucci E, Stampfer MJ, Colditz GA, et al: A comparison of prospective and retrospective assessments of diet in the study of breast. Am J Epidemiol 1993, 137:502-511.

27. Carroll RJ, Ruppert D, Stefanski LA: Measurement Error in Nonlinear Models. New York: Chapman and Hall, 1995.

28. Willett WC, Sampson L, Stampfer MJ, et al: Reproducibility and validity of a semiquantitative food frequency questionnaire. $\mathrm{Am} J$ Epidemiol 1985, 122:51-65.

29. Hebert JR, Clemow L, Pbert L, et al: Social desirability bias in dietary self-report may compromise the validity of dietary intake measures. Int J Epidemiol 1995, 24:389-398.

30. Hebert JR, Ma Y, Clemow L, et al: Gender differences in social desirability and social approval bias in dietary self-report. $A m \mathrm{~J}$ Epidemiol 1997, 146:1046-1055.

31. Heitmann BL, Lissner L: Dietary underreporting by obese individu- $\quad$ als: is it specific or non-specific. Br Med J 1995, 311:986-989.

A study of dietary self-reports of energy and protein consumption using objective measures of energy and protein expenditure is reported. It demonstrates a service under-reporting of energy among obsese persons. 
32. Steinmetz KA, Potter JD: Vegetables, fruit and cancer prevention: a review. J Am Diet Assoc 1996, 96:1027-1039.

33. Chlebowski RT, Blackburn GL, Buzzard IM, et al: Adherence to a dietary fat intake reduction program in postmenopausal women receiving therapy for early breast cancer. The Women's Intervention Nutrition Study. J Clin Oncol 1993, 11:2072-2080.

34. Pierce JP, Parker B, Wright F: A feasibility study for a randomized controlled trial of a plant-based diet on breast cancer recurrence [abstract]. Proc Am Soc Clin Oncol 1995, 14:160.

35. Boyd NF, Greenberg C, Lockwood G, et al: Effects at two years of a low fat, high carbohydrate diet on radiological features of the breast: results from a randomized trial. J Natl Cancer Inst 1997, 89:488-496.

36. Prentice RL, Sheppard L: Aggregate data studies of disease risk factors. Biometrika 1995, 82:113-125.

37. Sheppard L, Prentice RL: On the reliability and precision of withinand between-population estimates of relative risk parameters. Biometrics 1995, 51:853-863.

38. Fisher B, Constantino JP, Wickerham DL, et al: Tamoxifen for prevention of breast cancer: report of the National Surgical Adjuvant Breast and Bowel Project P-1 Study. J Natl Cancer Inst 1998, 90: 1371-1388.

39. Cummings RS, Eckert S, Krueger KA, et al: The effect of raloxifone on breast cancer risk in postmenopausal women. JAMA 1999, 281:2189-2197.

40. Taubes G: Epidemiology faces its limits. Science 1995, 269:164169.

Author's affiliation: Division of Public Health Sciences, Fred

Hutchinson Cancer Research Center, Seattle, Washington, USA

Correspondence: Ross L Prentice, Fred Hutchinson Cancer Research Center, 1100 Fairview Avenue North, Seattle, WA 98109, USA.

Tel: +1 206667 4267; fax: +1 206667 4142;

e-mail: rprentic@fhcrc.org 